\title{
Nivel de pobreza asociado al estadio de gravedad del cáncer ginecológico*
}

\author{
Relationship of gynaecologic cancer stage and poverty
}

\author{
César Gutiérrez ${ }^{1,2}$, Edith Alarcón ${ }^{1,3}$ \\ 1 Departamento Académico de Medicina Preventiva y Salud Pública, Facultad de Medicina, Universidad Nacional Mayor de San Marcos. Lima, Perú. \\ 2 Sección de Epidemiología, Instituto de Medicina Tropical Daniel A. Carrión, Facultad de Medicina, Universidad Nacional Mayor de San Marcos. Lima, Perú. \\ 3 Departamento de Estadística y Epidemiología del Cáncer, Instituto Nacional de Enfermedades Neoplásicas. Lima, Perú. \\ * El resumen del presente trabajo fue publicado en An Fac med. 2008;69 Supl 1:S33.
}

\begin{abstract}
Resumen
Objetivo: Determinar la relación entre el nivel de pobreza y el estadio al momento del diagnóstico de cáncer ginecológico. Diseño: Estudio ecológico de grupos múltiples. Lugar: Instituto Nacional de Enfermedades Neoplásicas (INEN). Participantes: Mujeres que buscaron atención por cáncer de cuello uterino y mama. Métodos: A partir del registro estadístico del INEN, para el quinquenio 2000-2004, se determinó la proporción de pacientes que buscaron atención por cáncer de cuello uterino y mama que llegaron en estadios avanzados (III y IV), provenientes de Lima y Callao. Para la determinación del nivel de pobreza del distrito de procedencia de las pacientes, se empleó el mapa de pobreza de Foncodes 2006, clasificando a los distritos de Lima y Callao en deciles, según el índice de carencias. Se calculó el coeficiente de correlación de Spearman para establecer la correlación entre la proporción de pacientes que presentaban un estadio avanzado de cáncer ginecológico y el nivel de pobreza. Principales medidas de resultados: Casos nuevos de cáncer de mama y de cuello uterino y nivel de pobreza. Resultados: En el período estudiado, se registró 2956 casos nuevos de cáncer de mama y 2547 de cáncer de cuello uterino. El 50,7\% de los casos de cáncer de mama y el $44,1 \%$ de cáncer de cuello uterino buscaron atención cuando ya tenían un estadio avanzado. Se estableció una correlación directa estadísticamente significativa entre el nivel de pobreza y casos avanzados de cáncer de mama ( $r h o=0,81, p<0,001$ ) y entre el nivel de pobreza y casos avanzados de cuello uterino (rho $=0,92, p<0,001$ ). Conclusiones: Se determinó una correlación directa fuerte entre el nivel de pobreza y casos avanzados de cáncer ginecológico, en pacientes atendidas en el INEN, en el periodo 2000-2004. Este podría ser un indicador de inequidad en el acceso a servicios de salud en la población de Lima y Callao.

Palabras clave: Neoplasias ginecológicas; neoplasias del cuello uterino; neoplasias de la mama; pobreza; inequidad en salud.
\end{abstract}

\begin{abstract}
Objective: To determine the relationship between poverty and stage at diagnosis of gynaecologic cancer. Design: Multiple groups ecologic study. Setting: Instituto Nacional de Enfermedades Neoplasicas (INEN - National Institute of (ancer). Participants: Women with both cervical and breast cancer. Methods: From 2000-2004 INEN statistics registry we determined the proportion of patients from Lima and Callao who sought care for both cervical and breast cancer advanced stages (III and IV). We registered the patients' district of origin poverty level by using the 2006 Foncodes poverty map, ranking the districts of Lima and Callao in deciles according to the deprivation index. The Spearman correlation coefficient was calculated to establish the proportion of patients with advanced stage of a gynaecologic cancer and the level of poverty. Main outcome measures: New cases of both breast and cervical cancer and poverty. Results: During the period studied there were 2956 new cases of breast cancer and 2547 new cases of cervical cancer; $50,7 \%$ of breast cancer and $44,1 \%$ of cervical cancer cases sought care with an advanced stage of the disease. A statistically significant direct correlation was found between poverty level and advanced cases of breast cancer (rho = $0,81, p<0,001)$ and between poverty and advanced cases of cervical cancer (rho $=0,92, p<0,001)$. Conclusions: We found a strong correlation between poverty level and advanced cases of gynaecologic cancer in patients attended at INEN in the period 2000-2004. This could be an indicator of access inequality to health services in the population of Lima and Callao.
\end{abstract}

Keywords: Gynaecologic neoplasms; uterine cervical neoplasms; breast neoplasms; poverty; health inequalities.

\section{INTRODUCCIÓN}

A nivel mundial, los diferentes tipos de cáncer son una de las principales causas de muerte. El año 2007 se registró un total de 7,9 millones de muertes por cáncer a nivel mundial, representando aproximadamente el $13 \%$ de todas las muertes. Cerca de $72 \%$ de las muertes por cáncer se producen en países de ingresos medios y bajos. Se estima que el número de muertes anuales por cáncer seguirá en aumento, proyectándose un incremento de $45 \%$ entre los años 2007 y 2030, llegando a 11,5 millones de muertes. Adicionalmente, 11,3 millones de personas fueron diagnosticadas de cáncer el año 2007, estimándose un incremento de casos nuevos anuales a 15,5 millones hacia el año $2030^{(1)}$. En
América Latina, se ha estimado para el año 2008 un total de 1011000 casos incidentes de cáncer, siendo más los casos en mujeres (522 000) que en hombres (489 000), y un total de 589000 muertes por cáncer. En los hombres, los tipos de cáncer más frecuentes son el de próstata, seguido del pulmonar, mientras que en las mujeres son el cáncer de mama seguido del de cuello uterino ${ }^{(2)}$.

En el Perú, el Instituto Nacional de Enfermedades Neoplásicas (INEN) muestra una tendencia ascendente del número de casos nuevos de cáncer diagnosticados anualmente; así, de 7326 casos diagnosticados el año 1997, se pasó a 8941 el año 2004, es decir un incremento de $22 \%$, en un periodo de ocho años ${ }^{(3,4)}$.
Si bien este incremento del número de casos puede deberse a una mejora en el sistema de referencias y en los métodos diagnósticos, nos indica también que las patologías oncológicas afectan cada vez con mayor frecuencia a la población peruana. Según estimaciones de la Organización Mundial de la Salud (OMS) ${ }^{(5)}$, el año 2005 fallecieron en el Perú 32000 personas por cáncer ( 88 muertes al día), lo que significa que $18,4 \%$ de muertes ese año en el país se debió a patologías oncológicas. Las mismas estimaciones proyectan en nuestro país que, hacia el año 2030, 22,9\% del total de defunciones serán por cáncer y representarán la segunda causa de muerte, después del grupo 'otras enfermedades crónicas'. 
Según datos del INEN, las neoplasias más frecuentemente diagnosticadas entre mujeres procedentes de las diferentes regiones del país son el cáncer de cuello uterino $(24,9 \%)$ y el cáncer de mama $(18,8 \%)^{(4)}$. Las tasas estandarizadas de incidencia estimada de cáncer a nivel nacional señalan que, el año 2002, por cada 100000 mujeres se presentaron 55 casos nuevos de cáncer de cuello uterino y 36 de mama; mientras que las tasas estandarizadas de mortalidad por cáncer a nivel nacional señalan que, el año 2005 por cada 100000 mujeres fallecieron 30 por cáncer de cuello uterino y 19 por cáncer de mama ${ }^{(5)}$. Según la Dirección General de Epidemiología del Ministerio de Salud, se observa a nivel nacional en el período 1998-2002 una tendencia estable de la tasa de mortalidad por cáncer de mama, pero con una leve disminución en los estratos no pobres y un leve aumento en los estratos pobres. En el caso del cáncer de cuello uterino, la tendencia de la tasa de mortalidad es ascendente, tanto en población pobre como no pobre ${ }^{(6)}$.

De acuerdo a las recomendaciones de la OMS, la prevención primaria y secundaria son fundamentales en la lucha contra las enfermedades neoplásicas, siendo muy importante la educación en salud que debe recibir la población, para reconocer la importancia de evitar exposiciones y hábitos de riesgo, así como tomar conciencia de la necesidad de evaluaciones médicas periódicas para un diagnóstico precoz ${ }^{(2)}$. En ese sentido, entre los objetivos planteados por la Coalición Multisectorial 'Perú Contra el Cáncer', se señala realizar tempranamente la detección del cáncer de cuello uterino y mama, entre otras patologías priorizadas ${ }^{(7)}$. Sin embargo, uno de los principales problemas en el manejo de las enfermedades neoplásicas es que el diagnóstico se hace en la mayoría de casos en estadios avanzados. En el INEN, 56,9\% del total de pacientes corresponde a estadios III o IV al momento del diagnóstico ${ }^{(4)}$; al diagnosticar a un gran porcentaje de pacientes en estadios avanzados, se espera que su supervivencia sea menor, que la discapacidad asociada a la enfermedad sea mayor, que la carga emocional para la paciente y su familia también se in- cremente y, además, que los gastos en la atención médica sean mayores.

El nivel socio económico es un factor estrechamente relacionado con el estado de salud. Se ha descrito en diversos estudios internacionales, tanto en países desarrollados como en vías de desarrollo, así como en estudios nacionales, que diferentes indicadores de pobreza están asociados a peores estados de salud y dificultades en el acceso a servicios de salud ${ }^{(8,9)}$. En este sentido, se ha demostrado que con una mayor cobertura y mejora en la capacidad diagnóstica y del personal de servicios de detección temprana de neoplasias en poblaciones de bajos recursos es posible lograr una mayor eficacia en el manejo de los pacientes con cáncer ${ }^{(10-12)}$.

Dada la compleja estructura social de nuestro país, donde en una región o provincia se pueden encontrar grupos poblaciones con marcadas diferencias en el nivel socio-económico, así como la desigual distribución de los recursos de salud pública que condiciona diferencias en la detección oportuna de las enfermedades oncológicas, el presente estudio tiene por objetivo determinar la relación entre el nivel de pobreza y el estadio con que las pacientes con cáncer ginecológico (cáncer de cuello uterino y cáncer de mama) acuden en busca de atención al Instituto Nacional de Enfermedades Neoplásicas (INEN).

\section{MÉTODOS}

El diseño de esta investigación corresponde a un estudio ecológico de grupos múltiples ${ }^{(13)}$. La unidad geográfica de análisis estuvo constituida por 43 distritos de Lima Metropolitana y 6 distritos de la región Callao. Para cada uno de los distritos se obtuvo información de las variables estadio de cáncer ginecológico de las pacientes que acudieron al INEN (nuevos diagnósticos) y pobreza, según el índice de carencias.

La variable estadio de cáncer ginecológico se obtuvo a partir del registro estadístico de las pacientes mujeres diagnosticadas de cáncer de cuello uterino (CIE10: C53) o de cáncer de mama (CIE10: C50) en el INEN, durante el quinquenio 2000-2004. Según el sistema de estadificación TNM, que está basado en la extensión del tumor (T), la diseminación a los ganglios linfáticos $(\mathrm{N})$, y la metástasis $(\mathrm{M})^{(14)}$, los tumores de los pacientes que acuden al INEN son clasificados en cuatro estadios por el médico especialista que realiza el diagnóstico, considerándose a los estadios III y IV neoplasias avanzadas. Para cada distrito, se determinó la proporción de pacientes procedentes de cada uno de ellos que presentaban un estadio avanzado al momento del diagnóstico. Debido a la relativa baja frecuencia absoluta de casos en algunos distritos (principalmente en los menos poblados), lo cual dificultaría el análisis con proporciones, es que se agrupó los distritos en deciles, según el nivel de pobreza.

El nivel de pobreza del distrito de procedencia de las pacientes se determinó mediante el índice de carencias, que presenta el Fondo Nacional de Cooperación para el Desarrollo Social (FONCODES) en el mapa de pobreza nacional, para el año $2006^{(15)}$. El índice de carencias es el resultado de la combinación de seis indicadores de pobreza, tres de carencias de servicios básicos (porcentaje de la población que carece de agua potable, porcentaje de la población que carece de desagüe o letrinas y porcentaje de la población que carece de electricidad) y tres de vulnerabilidad (porcentaje de mujeres analfabetas de 15 años y más, porcentaje de niños de 0 a 12 años de edad y porcentaje de niños con desnutrición crónica de 6 a 9 años). Este índice de carencias puede tomar valores entre 0 y 1 ; a mayor valor del indicador mayor pobreza del distrito. Los distritos de Lima Metropolitana y Callao fueron agrupados en deciles, para el índice de carencias (tabla 1).

El análisis estadístico fue del tipo descriptivo, en una primera etapa, calculándose posteriormente como medida de asociación entre el nivel de pobreza y la proporción de pacientes que presentaban un estadio avanzado de cáncer de cuello uterino o de mama, el coeficiente de correlación de Spearman. Se elaboró también gráficos de dispersión, para representar la asociación entre las variables estadio de cáncer ginecológico y nivel de pobreza. Para la tabulación de 
Tabla 1. Clasificación de los distritos de Lima Metropolitana y Callao según nivel de pobreza, en base a mapa de pobreza según Foncodes 2006.

\begin{tabular}{ccl}
\hline $\begin{array}{c}\text { Grupo de } \\
\text { pobreza }\end{array}$ & $\begin{array}{c}\text { Rango del índice } \\
\text { de carencias }\end{array}$ & \multicolumn{1}{c}{ Distritos } \\
\hline 1 & $0,0030-0,0040$ & Miraflores, San Isidro, La Punta, Jesús María \\
2 & $0,0042-0,0059$ & San Borja, Pueblo Libre, Lince, Magdalena del Mar, San Miguel \\
3 & $0,0060-0,0074$ & Barranco, Breña, Santiago de Surco, La Molina, Surquillo \\
4 & $0,0079-0,0126$ & La Perla, Bellavista, San Luis, Cercado de Lima, La Victoria \\
5 & $0,0140-0,0211$ & Los Olivos, Rímac, San Martín de Porres, Callao, Chaclacayo \\
6 & $0,0226-0,2680$ & $\begin{array}{l}\text { Santa Anita, Comas, Chorrillos, Independencia, Carmen de la } \\
\text { Legua Reynoso }\end{array}$ \\
7 & $0,0274-0,0443$ & $\begin{array}{l}\text { San Juan de Miraflores, El Agustino, San Juan de Lurigancho, } \\
\text { Villa María del Triunfo, Santa María del Mar }\end{array}$ \\
8 & $0,0450-0,0580$ & Villa el Salvador, Santa Rosa, Ate, Carabayllo, Lurigancho \\
9 & $0,0637-0,0940$ & Ancón, Ventanilla, Puente Piedra, Punta Hermosa, Lurín \\
10 & $0,1070-0,2008$ & San Bartolo, Cieneguilla, Punta Negra, Pucusana, Pachacamac \\
\hline
\end{tabular}

los datos, se utilizó el paquete estadístico SPSS 11,0.

\section{RESULTADOS}

En el período 2000-2004, se registraron 2956 casos nuevos de cáncer de mama y 2547 de cáncer de cuello uterino, entre mujeres que se atendieron en el INEN, procedentes de Lima Metropolitana y el Callao. Se pudo establecer el estadio de la patología en 49,1\% de los casos de cáncer de mama y en $91,3 \%$ de los casos de cáncer de cuello uterino. De las pacientes en las que se pudo determinar el estadio de la enfermedad, 50,7\% de los casos de cáncer de mama y $44,1 \%$ de cáncer de cuello uterino buscaron atención cuando ya tenían un estadio avanzado (figura 1 ).
Se estableció una correlación directa estadísticamente significativa entre el nivel de pobreza y casos avanzados de cáncer de mama, es decir que, a mayor nivel de pobreza, mayor la proporción de mujeres procedentes de Lima Metropolitana y Callao que acudían al INEN en estadios avanzados de esta patología (rho $=0,81 ; p<0,001$ ) (figura 2).

De la misma manera, se estableció una correlación directa estadísticamente significativa entre el nivel de pobreza y casos avanzados de cáncer de cuello uterino, es decir que, a mayor nivel de pobreza, mayor la proporción de mujeres procedentes de Lima Metropolitana y Callao que acudían al INEN en estadios avanzados de esta patología (rho=0,92; $p<0,001$ ) (figura 3).

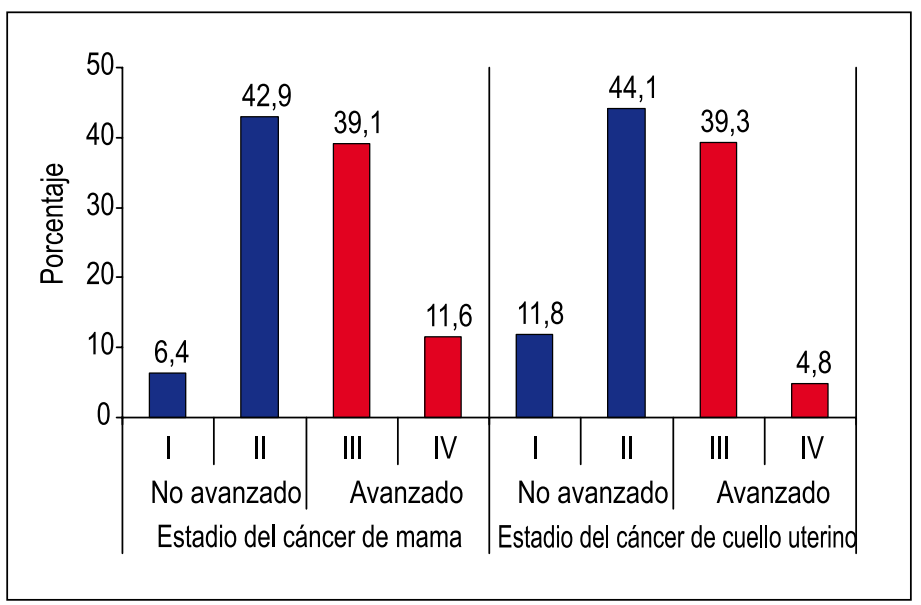

Figura 1. Estadio de enfermedad de los casos nuevos de cáncer ginecológico.

Para el decil más pobre, $66,7 \%$ de los casos de cáncer de mama y $62,5 \%$ de los casos de cáncer de cuello uterino fueron avanzados; mientras que para el decil menos pobre estas proporciones fueron $51,1 \%$ y $32,4 \%$, respectivamente. Al analizar las rectas de regresión lineal obtenidas, se observa que la proporción de casos de cáncer de mama diagnosticados en estadios avanzados se incrementa en $51 \%$ del decil de ingresos económicos mayores al decil más pobre, mientras que en el caso del cáncer de cuello uterino este incremento porcentual es de 65,2\%.

\section{DISCUSIÓN}

Los resultados del presente trabajo nos muestran que la pobreza se encuentra relacionada con una menor oportunidad de detección temprana de las neoplasias ginecológicas estudiadas. Ward y col. ${ }^{(16)}$ señalan que, en los EEUU, los habitantes de zonas pobres tienen $10 \%$ menos probabilidad de supervivencia a los cinco años de diagnosticado un cáncer, en comparación a zonas con mayores ingresos económicos. Así mismo, los casos de cáncer de mama y cuello uterino entre habitantes de zonas de mayores ingresos son en 33\% y $40,4 \%$ de extensión regional o con metástasis a distancia; mientras que, entre los habitantes de zonas pobres, estos porcentajes se elevan a $41 \%$ y $47,7 \%{ }^{(16)}$. Esta situación de disparidad en los EEUU, entre nivel socioeconómico y la morbilidad y mortalidad por cáncer de cuello uterino, se mantiene constante en el tiempo, a pesar de la tendencia descendente general de la incidencia y mortalidad ${ }^{(17)}$. Esto nos muestra que, si bien puede tenerse resultados en indicadores globales para un país, las brechas entre los diversos grupos socio-económicos deben formar parte del análisis de los indicadores.

En el Perú, se ha publicado estudios en los que se muestra las diferencias en el estado de salud y acceso a servicios médicos según estratos de pobreza, básicamente en salud materna e infantil ${ }^{(8,18)}$. Es interesante en este sentido el análisis realizado por la Organización Panamericana de la Salud, en base a los datos de la Encuesta de Hogares sobre Medición de Niveles de Vida (ENNIV), del 2000, donde se evidencia cómo los grupos poblacionales más 


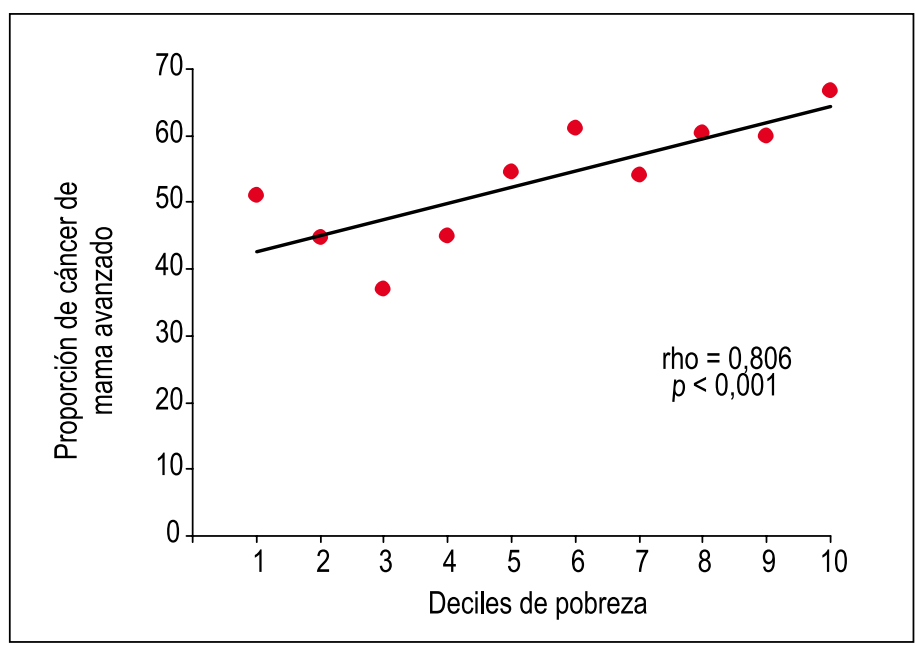

Figura 2. Relación entre el nivel de pobreza y estadios avanzados de cáncer de mama.

pobres presentan una menor búsqueda de atención por problemas de salud durante el último mes, independientemente de la edad, sexo o área de residencia (urbana o rural) ${ }^{(19)}$. Sin embargo, sobre enfermedades crónicas y en especial sobre cáncer ginecológico es poco lo publicado, desde una perspectiva de análisis de inequidad en salud según niveles de pobreza.

En el caso particular del cáncer de mama y cáncer de cuello uterino, lo que debe destacarse es que son neoplasias en las que puede detectarse tempranamente su aparición mediante métodos relativamente económicos. Para el cáncer de mama, un autoexamen bien realizado, un examen clínico por un profesional capacitado y una mamografía de rutina constituyen métodos de tamizaje con comprobada eficacia en países en vías de desarrollo; como lo es además el examen citológico con tinción Papanicolaou o la inspección visual del cérvix con ácido acético (IVA) o con solución yodoyodurada de lugol (IVSL), en el caso del cáncer de cuello uterino ${ }^{(1,2)}$. Sin embargo, la barrera del acceso a los servicios de salud sería un factor determinante para que las mujeres de estratos pobres no puedan acceder a estos métodos de prevención secundaria. Además, un componente fundamental en la prevención y control de estas patologías es la educación en salud, que está relacionada también con

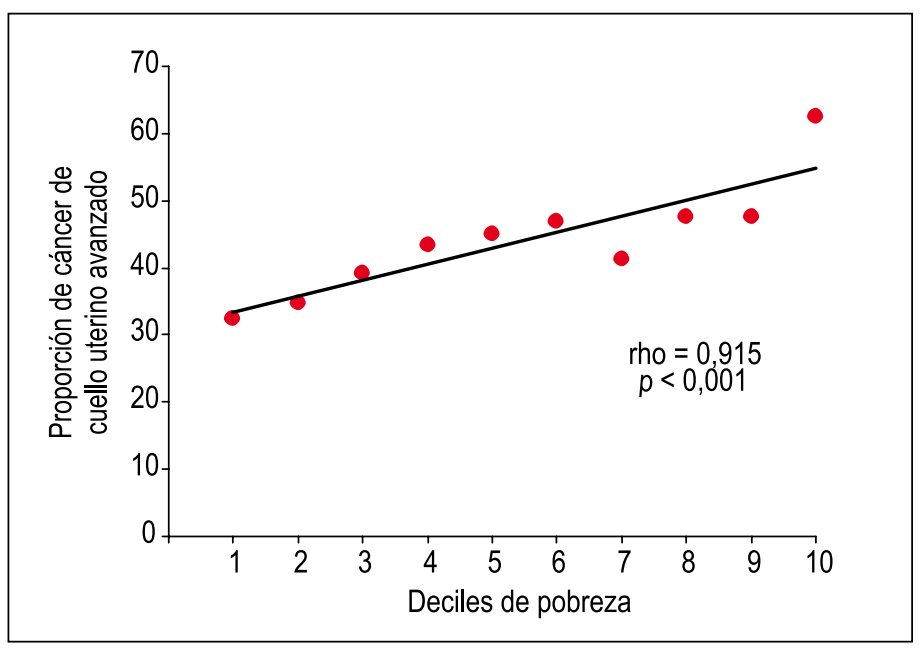

Figura 3. Relación entre el nivel de pobreza y estadios avanzados de cáncer de cuello uterino.

el acceso a los servicios y con el nivel socio-económico. A mayor nivel socioeconómico, mayor el conocimiento de la población sobre salud ${ }^{(20)}$. Las diferencias entre diferentes estratos socioeconómicos en las prácticas de diagnóstico precoz de cáncer ginecológico pueden reducirse cuando se promueve una participación activa de las mujeres, cuando las barreras culturales y económicas de acceso a servicios de salud son eliminadas y cuando se ofrece un adecuado soporte social ${ }^{(21)}$.

Nuestros resultados, al basarse solo en datos de las pacientes atendidas en un solo centro hospitalario, no pueden ser extrapolados a toda la población de Lima Metropolitana y la región Callao (a pesar de ser el INEN un centro de referencia). Además, podría haber una sub-estimación de la proporción de mujeres con casos avanzados de cáncer de mama, puesto que se tuvo el estadio solo en $49,1 \%$ de las pacientes. Por otro lado, siempre debe considerarse la posibilidad de ocurrencia de una falacia ecológica en este tipo de diseños ${ }^{(13)}$, por lo que debe tenerse cuidado al intentar extrapolar los resultados de un nivel poblacional (agregado) a un nivel individual. Además, debe considerarse también que la mayoría de los distritos presenta una composición heterogénea del nivel de pobreza entre sus habitantes. En el presente estudio, se ha trabajado con un indicador promedio, que podría esconder estas diferencias al interior de los agregados poblacionales. Sin embargo, los estudios ecológicos tienen la ventaja de obtener información de una forma rápida y económica; es más, si los registros continuos a partir de los cuales se elabora un estudio de este tipo son confiables y de calidad, los resultados obtenidos son sumamente útiles para la toma de decisiones en Salud Pública ${ }^{(22)}$.

Finalmente, concluimos que existe una asociación directa fuerte entre el nivel de pobreza y la proporción de casos avanzados de cáncer ginecológico en pacientes que fueron atendidas en el INEN, en el periodo 2000-2004, lo que constituiría un indicador de inequidad en el acceso a servicios y actividades de prevención primaria y secundaria de salud en la población femenina de Lima Metropolitana y la región Callao. 


\section{REFERENCIAS BIBLIOGRÁFICAS}

1. World Health Organization. Cancer [página web en Internet]. Geneva: WHO [citado el 27 de diciembre de 2008]. Disponible en: http://www. who.int/cancer/en/

2. Boyle P, Levin B (eds). World Cancer Report 2008. Lyon: International Agency for Research on Cancer; 2008.

3. Alarcón E, Ágreda F. Epidemiología descriptiva de neoplasias malignas diagnosticadas en el INEN (periodo 1997-2001). Boletín del INEN. 2004;26(1):11-32.

4. Poquioma E, Alarcón E. Epidemiología descriptiva de las neoplasias malignas en el INEN periodo 2000 2004. Boletín del INEN. 2007;29(2):66-81.

5. World Health Organization. WHO Global Infobase [base de datos en Internet]. Geneva: WHO [citado el 16 de junio de 2008]. Disponible en: http://www. who.int/infobase/report.aspx

6. Dirección General de Epidemiología. Análisis de la Situación de Salud del Perú - 2005. Lima: Ministerio de Salud del Perú; 2006.

7. Coalición Mutisectorial "Perú Contra el Cáncer". Plan Nacional para el fortalecimiento de la prevención y control del cáncer en el Perú. Lima: Coalición Mutisectorial "Perú Contra el Cáncer"; 2007.

8. Valdivia M. Acerca de la magnitud de la inequidad en salud en el Perú. Documento de Trabajo 37. Lima: GRADE; 2002.

9. Lama A. Determinantes del acceso a los servicios de salud en el Perú. Lima: Instituto Nacional de Estadística e Informática; 2000.

10.0'Malley A, Forrest C, Mandelblatt J. Adherence of low-income women to cancer screening recommendations. J Gen Intern Med. 2002;17(2):144-54.
11. Marchick J, Henson DE. Correlations between access to mammography and breast cancer stage at diagnosis. Cancer. 2005;103(8):1571-80.

12. O'Malley AS, Mandelblatt J, Gold K, Cagney KA, Kerner J. Continuity of care and the use of breast and cervical cancer screening services in a multiethnic community. Arch Intern Med. 1997;157(13):1462-70.

13. Morgenstern H. Ecologic Studies. En: Rothman K, Greenland S. Modern Epidemiology. 2da edición. Philadelfia: Lippincott Williams \& Wilkins; 1998. p. 459-80.

14. International Union Against Cancer. TNM classification of malignant tumours [página web en Internet]. Geneva: UICC [citado el 15 de diciembre de 2008]. Disponible en: http://www. uicc.org/index.php?option=com_content\&task= view\&id $=14275$ \&ltemid $=197$

15. Fondo de Cooperación para el Desarrollo Social. Mapa de pobreza 2006 [página web en Internet]. Lima: Foncodes [citado el 18 de agosto de 2008]. Disponible en: http://www.foncodes.gob. pe/mapapobreza/

16. Ward E, Jemal A, Cokkinides V, Singh GK, Cardinez C, Ghafoor A, et al. Cancer disparities by race/ ethnicity and socioeconomic status. CA Cancer J Clin. 2004;54(2):78-93.

17. Singh GK, Miller BA, Hankey BF, Edwards BK. Persistent area socioeconomic disparities in U.S. incidence of cervical cancer, mortality, stage, and survival, 1975-2000. Cancer. 2004;101(5):10517.

18. Pichihua J, Miranda M, Sánchez J, Trujillo H, Salvatierra R. Efecto del nivel socioeconómico sobre algunos indicadores de salud y nutrición en la niñez, Perú 2003 - 2004. Lima: Instituto Nacional de Salud; 2007.
19. Organización Panamericana de la Salud. Hoja Resumen sobre Desigualdades en salud. Perú [monografía en Internet]. Lima: Organización Panamericana de la Salud; 2002 [citado el 27 de diciembre de 2008]. Disponible en: http://www. paho.org/Spanish/HDP/HDD/hojaresumenperu. pdf

20. Trejo U, Granados JA, Ortiz L, Delgado G. Diferencias sociales de la detección oportuna de cáncer cérvico uterino en las mujeres trabajadoras de una universidad de la ciudad de México. Rev Esp Salud Publica. 2005;79(3):403-14.

21. Signan N. Socioeconomic status and cancer screening. En: Kogevinas M, Pearce N, Susser M, Boffetta P (eds). Social Inequalities and Cancer. Lyon: International Agency for Research on Cancer; 1997. p. $369-76$

22. Koepsell T, Weiss N. Epidemiologic Methods. New York: Oxford University Press; 2003. p. 281307.

Manuscrito recibido el 20 de noviembre de 2008 y aceptado para publicación el 22 de diciembre de 2008.

\section{Correspondencia:}

Dr. César Gutiérrez Villafuerte

Sección de Epidemiología, Instituto de Medicina Tropical Daniel A. Carrión - UNMSM

José Santos Chocano 199

Callao 02, Perú.

Correo-e: guti.1994@gmail.com 